\title{
Improving spectral validation rates in hydrogen- deuterium exchange data analysis
}

\author{
Shaunak Raval ${ }^{1}$, Vladimir Sarpe ${ }^{2}$, Morgan Hepburn ${ }^{2}$, Alex Crowder ${ }^{2}$, Terry Zhang ${ }^{3}$, Rosa
} Viner $^{3}$, David Schriemer ${ }^{1,2}$

${ }^{1}$ Department of Chemistry, University of Calgary, Calgary, Alberta, Canada T2N-4N1

${ }^{2}$ Department of Biochemistry and Molecular Biology, University of Calgary, Alberta, Canada, T2N-4N1

${ }^{3}$ Thermo Fisher Scientific, 355 River Oaks Parkway, San Jose, California 95134, United States

*Corresponding Author: dschriem@ucalgary.ca

Dr. David Schriemer

Department of Biochemistry and Molecular Biology, Cumming School of Medicine

University of Calgary, 3330 Hospital Drive NW T2N-4N1

Supplementary Figure 


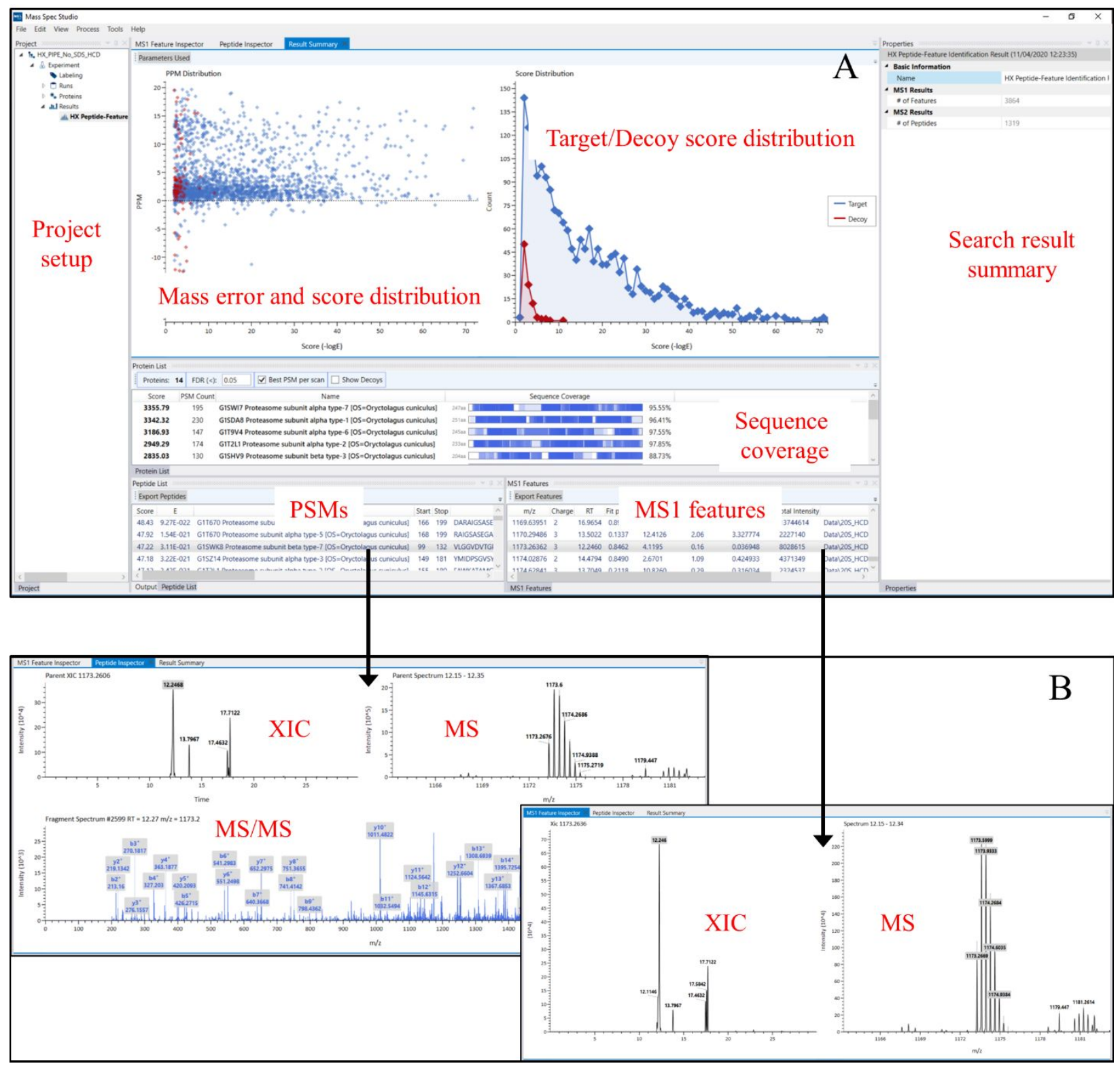

Figure S1. Screenshots of HX-PIPE application, showing (A) results of the MS/MS data search with respect to mass accuracy and false positive rates and (B) spectra data associated with individual entries in the peptide and MS1 feature list. 

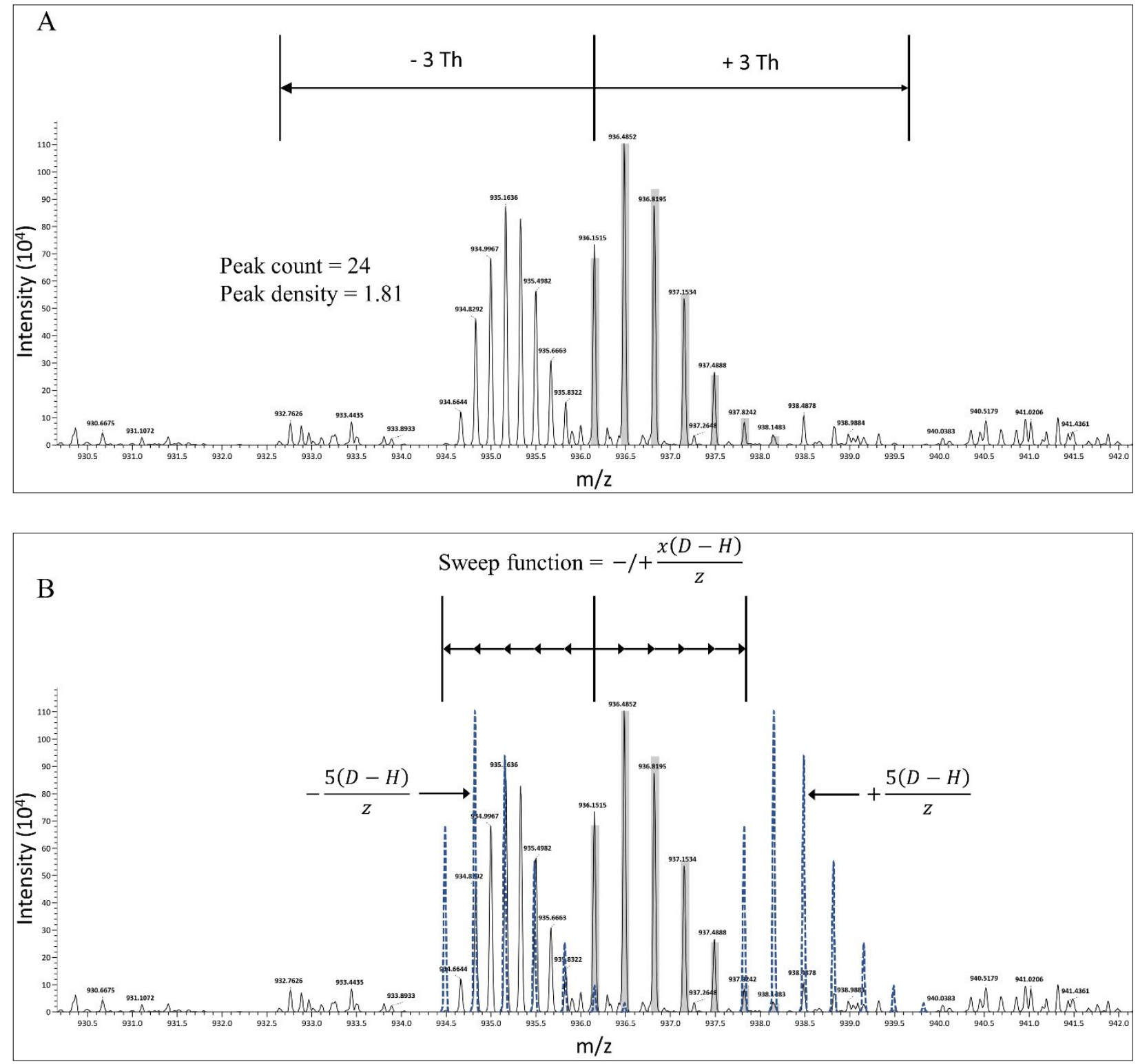

Figure S2. Strategies for estimating complexity and peak overlap. (A) the peak density method and (B) the sweep function method. Ranges shown for illustration only. 


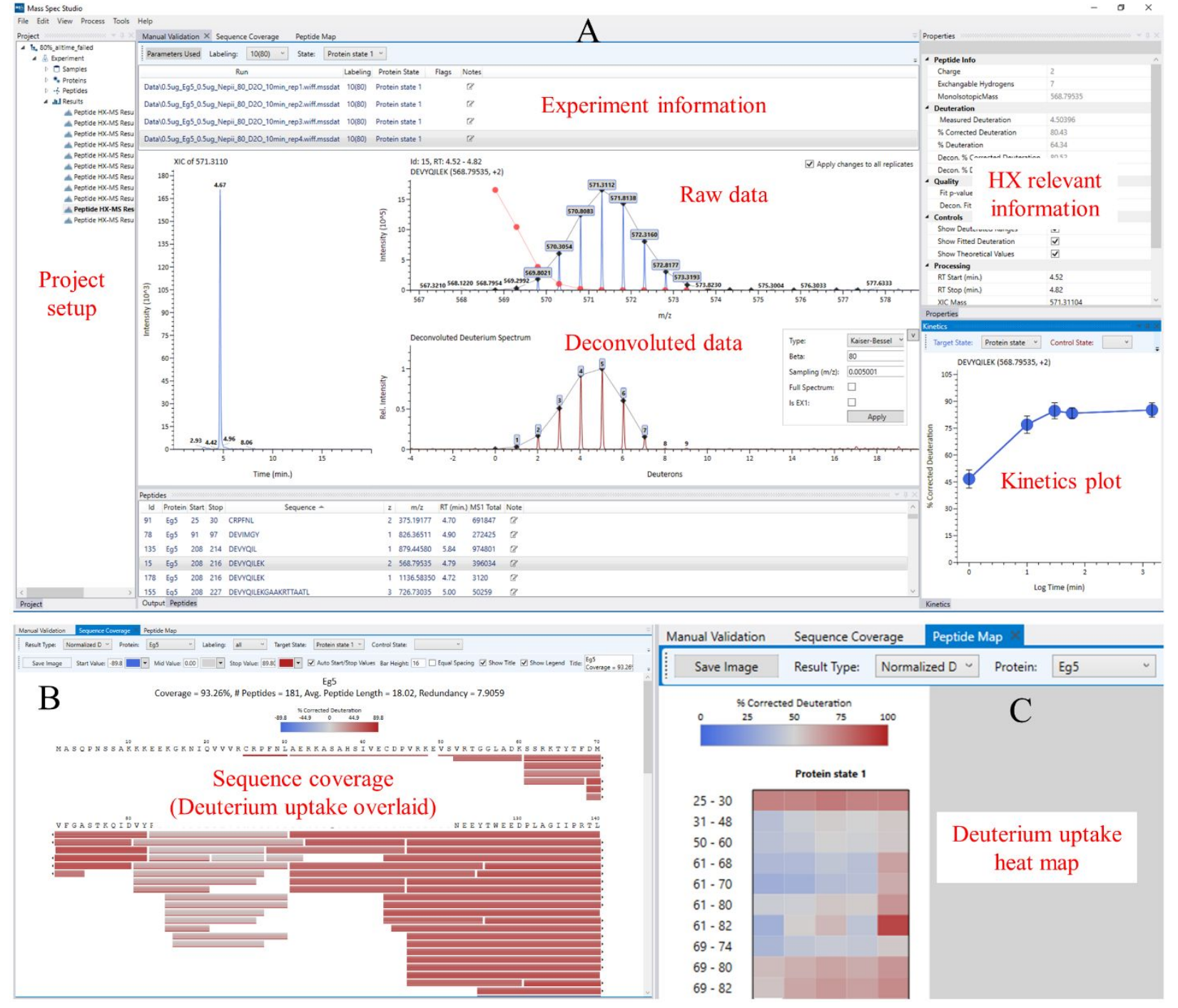

Figure S3. Processing features in HX-DEAL 


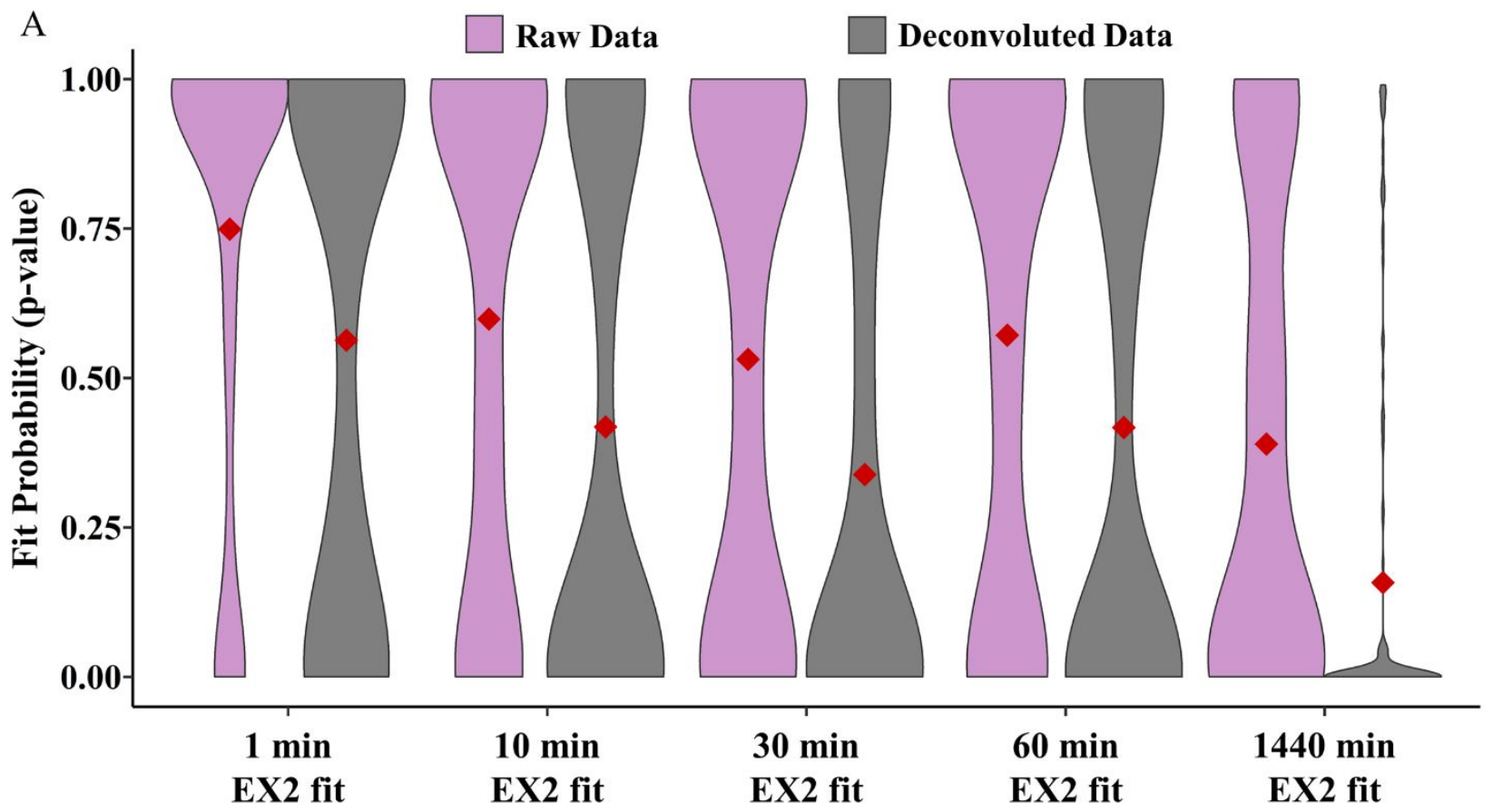

B

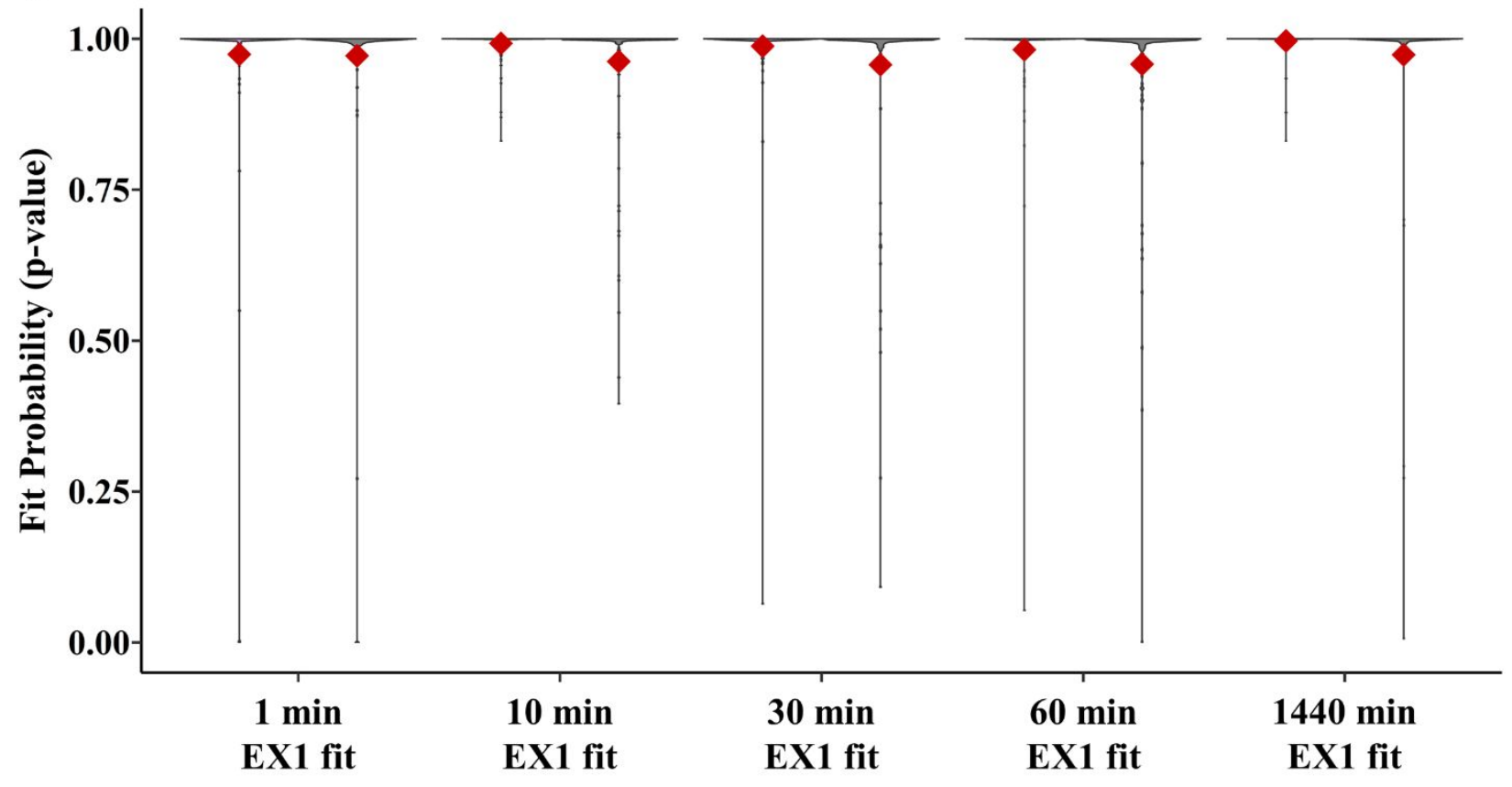

Figure S4. Violin plots showing the deuterium distribution analysis of the $20-80 \%$ sample blend, as per figure 7 (main text), as a function of labeling time. (A) Using CalcDeut to fit an EX2 model. (B) Using CalcDeut to fit an EX1 model. 
Table S1. Parameters for configuring an HX-PIPE search and an HX-DEAL data extraction for Eg5 and the 20S proteasome.

Table S2. Peptide-level classification of Eg5 HX-MS data and 20S proteasome HX-MS data, according to useability across all timepoints. 